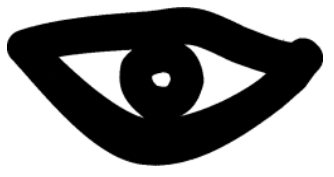

\title{
Enfoques de trabajo e implicación en los deberes escolares en estudiantes de Educación Primaria
}

Sara Rodríguez-Pereiro, Bibiana Regueiro, Susana Rodríguez, Isabel Piñeiro, Irene Pan, Benigno Sánchez y Antonio Valle

Universidade da Coruña

\begin{abstract}
Resumen
El objetivo de este trabajo es analizar las relaciones entre el rendimiento académico y la implicación de los estudiantes y el enfoque de trabajo que utilizan al hacer los deberes. La muestra es de 180 estudiantes de Educación Primaria. Los resultados indican que los niveles de rendimiento más altos se asocian a una mayor cantidad de deberes realizados, a un mejor aprovechamiento del tiempo y a una utilización de un enfoque profundo al hacer esos deberes. Según van avanzando de curso, los estudiantes dedican más tiempo, aprovechan menos ese tiempo y adoptan un enfoque más superficial al hacer los deberes.

Palabras clave: enfoques de trabajo en los deberes escolares, implicación del estudiante en los deberes escolares, Educación Primaria.
\end{abstract}

\section{Abstract}

The aim of this paper is to analyze the relationship between academic achievement and the students' involvement and the working approach used to do homework. The sample is 180 students of primary education. The results indicate that higher achievement levels are associated with a higher amount homework done, a better use of time and a use of a deeper approach to do homework. According advancing course, students spend more time, but the time management is worse and they adopt a more superficial approach to homework too Keywords: Approaches to homework, student homework engagement, Primary education.

\section{Introducción}

Algunos autores (Cooper, Robinson y Patall, 2006) consideran los deberes escolares como una parte más del trabajo académico del alumnado, que se asignan para realizar fuera del horario habitual de clases con el fin de extender y ampliar la práctica de habilidades académicas. Para muchos expertos, la realización de deberes contribuye a desarrollar unos buenos hábitos de estudio y promueven una mayor independencia y responsabilidad en el proceso de aprendizaje. Al mismo tiempo, los padres conocen más de cerca lo que aprenden sus hijos e hijas en la escuela y, de este modo, también se estrechan los vínculos familia-escuela (Epstein y Van Voorhis, 2001). Zimmerman y Kitsantas (2005) examinaron la importancia de la autoeficacia y de completar los deberes en relación con el rendimiento académico. Sin embargo, existen corrientes que opinan que son una sobrecarga de trabajo excesiva para los alumnos y alumnas así como una fuente importante de estrés y preocupación a la vez que una fuente que provoca un incremento de las desigualdades sociales en el aula.

Hay que señalar que los resultados de la investigación sobre el efecto de los deberes en los resultados académicos de los estudiantes son algo inconsistentes, especialmente en Educación Primaria. Por eso, el principal propósito de este trabajo es, en primer lugar, conocer las diferencias que existen en las variables vinculadas con la implicación en los deberes escolares (cantidad de deberes realizados de los prescritos por el profesor, cantidad de tiempo utilizado en dicha realización y aprovechamiento de ese tiempo) y en el enfoque de trabajo al hacer los deberes (profundo $\mathrm{y}$ superficial) en función de los distintos niveles de rendimiento académico (insuficiente, suficiente, bien, notable y sobresaliente) de los estudiantes de $4^{\circ}, 5^{\circ}$ y $6^{\circ}$ de Educación Primaria. Debido al posible efecto que pueden tener en esta relación el género y el curso, se controló dicho efecto incorporándolas como covariables. En segundo lugar, y en caso de que las covariables sean significativas, se pretende conocer en qué sentido se producen los cambios en las variables utilizadas en el estudio.

Como posibles hipótesis, y teniendo en cuenta que parece haber una relación positiva entre la cantidad de deberes realizados (de los prescritos por el profesorado) y el rendimiento académico, sobre todo en estudiantes de Secundaria y de Bachillerato (Cooper et al., 2006), aunque también en estudiantes de Primaria (Pan et al., 2013), se espera encontrar que aquellos estudiantes con los niveles más altos de rendimiento académico sean los que realizan una mayor cantidad de deberes. Al mismo tiempo, se espera encontrar que los niveles más altos de rendimiento académico estén asociados con un mejor aprovechamiento del tiempo dedicado a los deberes y con el empleo de menos tiempo en realizar esos deberes. Además, también esperamos obtener que aquellos estudiantes con los niveles más altos de rendimiento adopten más un enfoque de tipo profundo y menos de tipo superficial al realizar los deberes.

\section{Método}

\section{Participantes}

En el estudio participaron 180 estudiantes pertenecientes a cuatro centros públicos de Educación Primaria de la provincia de A Coruña. El 49.4\% ( $\mathrm{N}=$ $89)$ son chicos y el $50.6 \%(\mathrm{~N}=91)$ son chicas. De edades comprendidas entre los 9 y los 13 años, el $41.7 \%$ $(\mathrm{N}=75)$ cursaban $4^{\circ}$ curso de Educación Primaria, el $34.4 \%(\mathrm{~N}=62)$ cursaban $5^{\circ}$ curso de Educación Primaria y el $23.9 \%(\mathrm{~N}=43)$ cursaban $6^{\circ}$ curso de Educación Primaria.

\section{Instrumentos}

- Implicación en los deberes escolares. Para medir el tiempo dedicado a los deberes, el aprovechamiento

Agradecimientos: Este trabajo se ha desarrollado gracias a la financiación del proyecto de investigación EDU2013-44062-P, perteneciente al Plan Estatal de Investigación Científica y Técnica y de Innovación 2013-2016 (MINECO) y al financiamiento recibido por una de las autoras en el Programa FPU del Ministerio de Educación, Cultura y Deporte.

Correspondencia: Antonio Valle.vallar@udc.es

Selección y peer-review bajo responsabilidad del Grupo de Investigación G000422-GIPDAE, Universidade da Coruña, España. 
del tiempo y la cantidad de deberes realizados se utilizó la Encuesta sobre los Deberes Escolares $(E D E)$, la cual fue utilizada en estudios recientes (p.e., Núñez et al., 2013;Núñez et al., 2015; Rosário et al., 2009; Valle et al., 2015) para la obtención de este tipo de datos.

- Enfoque de trabajo al hacer los deberes escolares. Para medir el proceso o modo de trabajo en los deberes escolares, se realizó una adaptación del Students' Approaches to Learning Inventory (Rosário et al., 2010, 2013), teniendo en cuenta tanto la edad de los alumnos como el contextos de los deberes escolares. Este instrumento está basado en la investigación existente en el campo de los enfoques de aprendizaje y estudio y aporta información sobre dos modos, o enfoques, de acercarse al trabajo sobre los deberes escolares: superficial y profundo

- Rendimiento académico. La evaluación del rendimiento académico se obtuvo mediante las calificaciones académicas finales obtenidas por los estudiantes en el curso anterior en las materias de Lengua Española, Lengua Gallega, Lengua Inglesa, Conocimiento del Medio y Matemáticas. Y el rendimiento medio se calculó a través del promedio de las calificaciones en las cinco materias mencionadas.

\section{Procedimiento}

Los datos referidos a las variables objeto de estudio fueron recogidos durante el horario escolar, previo consentimiento del equipo directivo y de los profesores de los alumnos.

\section{Análisis de datos}

Con la finalidad de dar respuesta a los objetivos del trabajo, se realizó un MANCOVA, tomando como factor el rendimiento académico de los estudiantes (con cinco niveles) y como variables dependientes la implicación en los deberes escolares (cantidad de deberes realizados de los prescritos por el profesor, cantidad de tiempo utilizado en dicha realización y aprovechamiento de ese tiempo) y el enfoque de trabajo al hacer los deberes (profundo y superficial). Se incluyen como covariables el género y el curso para explorar su potencial papel modulador.

Posteriormente, en caso de que esas covariables sean significativas, se realizará un Análisis Multivariado de Varianza (MANOVA) tomando el género y el curso como variables independientes y así conocer en qué sentido se producen los cambios en las variables dependientes (implicación en los deberes y enfoques de trabajo al hacer los deberes). Para medir el tamaño del efecto se ha utilizado el coeficiente eta-cuadrado parcial (१р2), ya que es uno de los más utilizados habitualmente dentro de la investigación educativa (ver, por ejemplo, Sun, Pan y Wang, 2010). Para la interpretación de los tamaños del efecto se utilizó el criterio establecido en el trabajo clásico de Cohen (1988), en base al cual, un efecto es pequeño cuando $\eta \mathrm{p} 2=.01(d=.20)$, el efecto es medio cuando $\eta \mathrm{p} 2=$ $.059(d=.50)$ y el tamaño del efecto es grande si $\eta p 2=$ $.138(d=.80)$.

\section{Resultados}

Diferencias en la implicación en los deberes y en los enfoques de trabajo en función del nivel de rendimiento académico (controlando el efecto del curso y del género)

Una vez controlado el efecto de la variable curso $\left(\lambda_{\text {Wilks }}=.883, F(5,169)=4.47 ; p<.01, \eta_{\mathrm{p}}{ }^{2}=.117\right) \mathrm{y} \mathrm{de}$ la variable género $\left(\lambda_{\text {Wilks }}=.965, F(5,169)=1.23 ; p=\right.$ $.29, \eta_{\mathrm{p}}{ }^{2}=.035$, los resultados indican que hay diferencias estadísticamente significativas en el conjunto de variables dependientes estudiadas en función de los distintos niveles de rendimiento académico $\left(\lambda_{\text {Wilks }}=.728, F(20,561)=2.82 ; p<.001, \eta_{\mathrm{p}}{ }^{2}\right.$ $=.076)$. El tamaño del efecto es medio.

Teniendo en cuenta los datos referidos a cada variable dependiente considerada individualmente, hay diferencias estadísticamente significativas en función del nivel de rendimiento académico de los estudiantes en la cantidad de deberes realizados $(\mathrm{F}(4,173)=7.29$, $\left.\mathrm{p}<.001 ; \eta_{\mathrm{p}}{ }^{2}=.144\right)$, en el aprovechamiento del tiempo dedicado a los deberes $\left(\mathrm{F}(4,173)=4.54, \mathrm{p}<.01 ; \eta_{\mathrm{p}}{ }^{2}=\right.$ $.095)$ y en el enfoque superficial $(\mathrm{F}(4,173)=5.50$, $\left.\mathrm{p}<.001 ; \eta_{\mathrm{p}}{ }^{2}=.113\right)$. En todos los casos, los tamaños del efecto son grandes o próximos a grandes.

Diferencias en la implicación en los deberes y en los enfoques de trabajo en función curso

Los resultados del MANOVA (tomando como factor el curso), llevado a cabo para conocer con detalle hasta qué punto influyen el curso en cada una de las variables dependientes (cantidad de deberes realizados, tiempo dedicado a los deberes, aprovechamiento del tiempo y tipo de enfoque de trabajo utilizado: superficial o profundo), indican que de forma general en el conjunto de variables estudiadas sí hay diferencias estadísticamente significativas en función del curso $\left.\lambda_{\text {Wilks }}=.887, F(10,346)=3.22 ; p<.01, \eta_{\mathrm{p}}{ }^{2}=.085\right) . \mathrm{El}$ tamaño del efecto es medio.

Concretamente y teniendo en cuenta los datos referidos a cada variable dependiente considerada individualmente, hay diferencias estadísticamente significativas en función del curso en cuanto al aprovechamiento del tiempo dedicado a los deberes $\left(\mathrm{F}(2,177)=6.30, \mathrm{p}<.01 ; \eta_{\mathrm{p}}{ }^{2}=.066\right)$ y el enfoque profundo $\left(\mathrm{F}(2,177)=9.05, \mathrm{p}<.001 ; \eta \mathrm{p}^{2}=.093\right)$. Los tamaños del efecto son medios.

\section{Discusión}

Los resultados indican que hay un aumento progresivo en la cantidad de deberes realizados y en el aprovechamiento del tiempo dedicado a los deberes así como una mayor utilización del enfoque profundo a medida que los niveles de rendimiento académico son más altos.

Otro resultado destacado es que según van avanzando los estudiantes de $4^{\circ}$ a $6^{\circ}$ curso de Primaria se produce un aumento progresivo del tiempo que dedican a hacer los deberes escolares $y$, sin embargo, el aprovechamiento que hacen de ese tiempo va siendo cada vez menor. Esta disminución del aprovechamiento del tiempo dedicado a los deberes coincide con los resultados de otros trabajos realizados con estudiantes de Primaria (p.e., Valle et al., 2015), donde se observa 
una disminución progresiva desde del aprovechamiento del tiempo a medida que los estudiantes avanzan de curso.

Los resultados encontrados en este trabajo parecen demostrar que cuanto mayor es el rendimiento también aumenta la cantidad de deberes escolares que realizan los estudiantes (de los prescritos por el profesorado) y el aprovechamiento del tiempo que dedican a realizar esos deberes. Sin embargo, esta relación positiva y lineal encontrada entre el rendimiento académico con la cantidad de deberes y el aprovechamiento del tiempo no parece mantenerse con respecto al tiempo dedicado a los deberes. Probablemente, aquellos estudiantes que tienen un rendimiento más alto necesitan menos tiempo porque tienen más habilidades y conocimientos previos o porque gestionan mejor el tiempo que dedican a los deberes (o ambas cosas a la vez) que aquellos estudiantes que tiene un rendimiento más bajo.

También, a nivel de curso, hemos encontrado que, a medida que avanza el alumnado de curso, el tipo de enfoque de trabajo que utilizan al realizar los deberes escolares tiende a ser cada vez más superficial. Estos datos son bastante preocupantes si estamos diciendo que con la edad los alumnos aprovechan menos el tiempo y se implican de una manera más superficial llevando a cabo estrategias menos autorreguladas y teniendo una motivación más extrínseca que intrínseca por el aprendizaje. Algunos estudios ratifican también estos mismos resultados (Regueiro et al., 2015).

Se puede deducir de aquí que a medida que el alumnado avanza en edad asocia el estudio, en este caso, la realización de los deberes escolares, con tareas escasamente significativas, poco útiles $\mathrm{y}$ poco motivantes. El alumnado al no ver utilidad en las mismas, lleva a cabo un tipo de enfoque más superficial con la única pretensión de aprobar las asignaturas, pero sin pararse a desmenuzar lo que está aprendiendo de manera profunda y utilizando estrategias que impliquen reflexión, interpretación y razonamiento. De ahí, también podemos deducir que el aprovechamiento del tiempo a la hora de realizar los deberes disminuya al no sentirse motivados intrínsecamente por la tarea a realizar.

Por tanto, según los resultados de este trabajo, los niveles más altos de rendimiento académico se asocian con una mayor cantidad de deberes realizados y con un mejor aprovechamiento del tiempo dedicado estos deberes, pero también con una menor utilización de un enfoque superficial al hacer los deberes. La mayor parte de las investigaciones muestran una relación positiva entre la cantidad de deberes realizados y el rendimiento académico, especialmente en estudiantes de Secundaria y Bachillerato (Cooper et al., 2006), pero también en estudiantes de Primaria (Pan et al., 2013; Valle et al., 2015). En base a estos resultados, se puede afirmar que, de modo general, "hacer los deberes lleva consigo que el rendimiento académico mejore" (Cooper et al, 2006, pág. 48).

De todos modos, aunque la cantidad de deberes realizados por los estudiantes (de los prescritos por sus profesores) es una variable que se asocia positivamente con el rendimiento académico, no se debe perder de vista que más allá de la cantidad de deberes realizados también es necesario tener en cuenta cómo es la calidad del proceso de realización de los deberes y los beneficios que genera en el propio proceso de construcción de conocimientos de los estudiantes.

\section{Referencias}

Cohen, J. (1988). Statistical power analysis for the behavioral sciences ( $2^{\mathrm{a}}$ ed.).Hillsdale,

NJ: Erlbaum.

Cooper, H., Robinson, J. y Patall, E. (2006). Does homework improve academic achievement? A synthesis of research, 1987-2003. Review of Educational Research, 76, 1-62.

Epstein, J. L. y Van Voorhis, F. L. (2001). More Than Minutes: Teachers' Roles in Designing Homework. Educational Psychologist, 36(3), 181-193.

Núñez, J. C., Suárez, N., Cerezo, R., González-Pienda, J. A., Rosário, P., Mourao, R. y Valle, A. (2013). Homework and academic achievement across Spanish Compulsory Education. Educational Psychology. doi: 10.1080/01443410.2013.817537

Núñez, J. C., Suárez, N., Rosário, P., Vallejo, G., Cerezo, R. y Valle, A. (2015). Teachers' feedback on homework, homework-related behaviors and academic achievement. The Journal of Educational Research, 108(3), 204-216.

Pan, I., Regueiro, B., Ponte, B., Rodríguez, S., Piñeiro, I. y Valle, A. (2013). Motivación, implicación en los deberes escolares y rendimiento académico. Aula Abierta, 41(3), 13-22.

Regueiro, B., Suárez. N., Valle, A., Núñez, J.C. y Rosário, P. (2015). La motivación e implicación en los deberes escolares a lo largo de la escolaridad obligatoria. Revista de Psicodidáctica, 20(1), 47-63.

Rosário, P., Mourão, R., Baldaque, M., Nunes, T., Núñez, J.C., González-Pienda, J.A., Cerezo, R. y Valle, A. (2009). Tareas para casa, autorregulación del aprendizaje y rendimiento en matemáticas. Revista de Psicodidáctica, 14, 179-192.

Rosário, P., Núñez, J. C., Fernando, P. J., Paiva, M. O., Lourenço, A., Cerezo, R. y Valle, A. (2013). The relationship between approaches to teaching and approaches to studying: a two-level structural equation model for biology achievement in high school. Metacognition and Learning, 8, 47-77.

Rosário, P., Núñez, J. C., González-Pienda, J. A., Valle, A., Trigo, L. y Guimarães, C. (2010). Enhancing selfregulation and approaches to learning in first-year college students: A narrative-based program assessed in the Iberian Peninsula. European Journal of Psychology of Education, 25, 411-428.

Sun, Sh., Pan, W. y Wang, L. L. (2010). A comprehensive review of effect size reporting and interpreting practices in academic journals in education and psychology. Journal of Educational Psychology, 102, 989-1004.

Valle, A., Pan, I., Núñez, J. C., Rosário, P., Rodríguez, S. y Regueiro, B. (2015). Deberes escolares y rendimiento académico en Educación Primaria. Anales de Psicología, 31(2), 562-569.

Zimmerman, B. y Kitsantas, A. (2005). Homework practices and academic achievement: The mediating role of self-efficacy and perceived responsibility beliefs. Contemporary Educational Psychology, 30(4), 397-417. 